\title{
An absolute calibration of DENIS (deep near infrared southern sky survey)
}

\author{
P. Fouqué ${ }^{1,2}$, L. Chevallier ${ }^{2}$, M. Cohen ${ }^{3}$, E. Galliano ${ }^{2}$, C. Loup $^{4}$, C. Alard ${ }^{15}$, B. de Batz ${ }^{5}$ E. Bertin ${ }^{4}$, \\ J. Borsenberger ${ }^{4}$, M.R. Cioni ${ }^{6}$, E. Copet $^{1}$, M. Dennefeld ${ }^{4}$, S. Derriere ${ }^{7}$, E. Deul ${ }^{6}$, P.-A. Duc ${ }^{8}$, D. Egret $^{7}$, \\ N. Epchtein ${ }^{9}$, T. Forveille ${ }^{10}$, F. Garzón ${ }^{16}$, H.J. Habing ${ }^{6}$, J. Hron ${ }^{11}$, S. Kimeswenger ${ }^{12}$, F. Lacombe ${ }^{1}$, \\ T. Le Bertre ${ }^{13}$, G.A. Mamon ${ }^{4,14}$, A. Omont ${ }^{4}$, G. Paturel ${ }^{17}$, S. Pau ${ }^{1}$, P. Persi ${ }^{18}$, A.C. Robin ${ }^{19}$, D. Rouan $^{1}$, \\ M. Schultheis ${ }^{4}$, G. Simon ${ }^{15}$, D. Tiphène ${ }^{1}$, I. Vauglin ${ }^{17}$, and S.J. Wagner $^{20}$ \\ 1 DESPA, Observatoire de Paris, 5 place J. Janssen, F-92195 Meudon Cedex, France \\ 2 European Southern Observatory, Casilla 19001, Santiago 19, Chile \\ 3 Radio Astronomy Laboratory, 601 Campbell Hall, University of California, Berkeley, CA 94720, U.S.A. \\ 4 Institut d'Astrophysique de Paris, 98 bis Bd. Arago, F-75014 Paris, France \\ 5 DASGAL, Observatoire de Paris, 5 place J. Janssen, F-92195 Meudon Cedex, France \\ ${ }^{6}$ Leiden Observatory, University of Leiden, P.O. Box 9513, 2300 RA Leiden, The Netherlands \\ 7 CDS, Observatoire Astronomique de Strasbourg, UMR 7550, 11 rue de l'Université, F-67000 Strasbourg, France \\ 8 CEA, DSM, DAPNIA, Centre d'Études de Saclay, F-91191 Gif-sur-Yvette Cedex, France \\ ${ }^{9}$ Observatoire de la Côte d'Azur, Département Fresnel, F-06304 Nice Cedex, France \\ 10 Observatoire de Grenoble, 414 rue de la Piscine, Domaine Universitaire de Saint Martin d'Hères, F-38041 Grenoble, France \\ 11 Institut für Astronomie der Universität Wien, Türkenschanzstrasse 17, A-1180 Wien, Austria \\ 12 Institut für Astronomie, Innsbruck University, A-6020 Innsbruck, Austria \\ 13 DEMIRM, Observatoire de Paris, 61 Av. de l'Observatoire, F-75014 Paris, France \\ 14 DAEC, Observatoire de Paris, 5 place J. Janssen, F-92195 Meudon Cedex, France \\ 15 DASGAL, Observatoire de Paris, 61 Av. de l'Observatoire, F-75014 Paris, France \\ 16 Instituto de Astrofísica de Canarias, E-38200 La Laguna, Tenerife, Spain \\ 17 CRAL, Observatoire de Lyon, F-69561 Saint-Genis Laval Cedex, France \\ 18 Istituto di Astrofisica Spaziale, CNR, C.P. 67, I-00044 Frascati, Italy \\ 19 Observatoire de Besançon, BP. 1615, F-25010 Besançon Cedex, France \\ 20 Landessternwarte Heidelberg, Königstuhl, D-69117 Heidelberg, Germany \\ e-mail: pfouque@eso.org
}

Received June 24; accepted October 20, 1999

\begin{abstract}
An absolute calibration of the DENIS photometric system is presented. It includes the determination of the overall transmission profiles in the 3 bands, namely $i, J$ and $K_{\mathrm{s}}$, combining contributions from atmosphere, telescope mirrors, instrument lenses and dichroics, filters, and detectors. From these normalized profiles, isophotal and effective wavelengths are computed, using the same synthetic Vega spectrum as that used to support the absolute calibration of many other ground-based and spaceborne photometric systems. Flux densities at zero magnitude are derived and integrated to give in-band fluxes, which are used to compute theoretical zero-points and compare them to observed ones, yielding estimates of the overall throughput of the whole system.
\end{abstract}

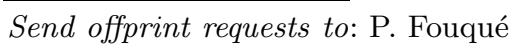

Key words: surveys — infrared: general instrumentation: miscellaneous

\section{Introduction}

The main goal of the DENIS survey (Deep Near-Infrared Southern Sky Survey, see Epchtein et al. (1994) for a complete introduction to DENIS) is to bridge the gap between the optical surveys on Schmidt plates and the far-infrared IRAS survey. Many aspects of astrophysics will benefit from such a survey, particularly studies of cool stars and heavily obscured regions.

Each night, roughly 1 million stars are detected by at least one of the 3 cameras of the DENIS instrument. 


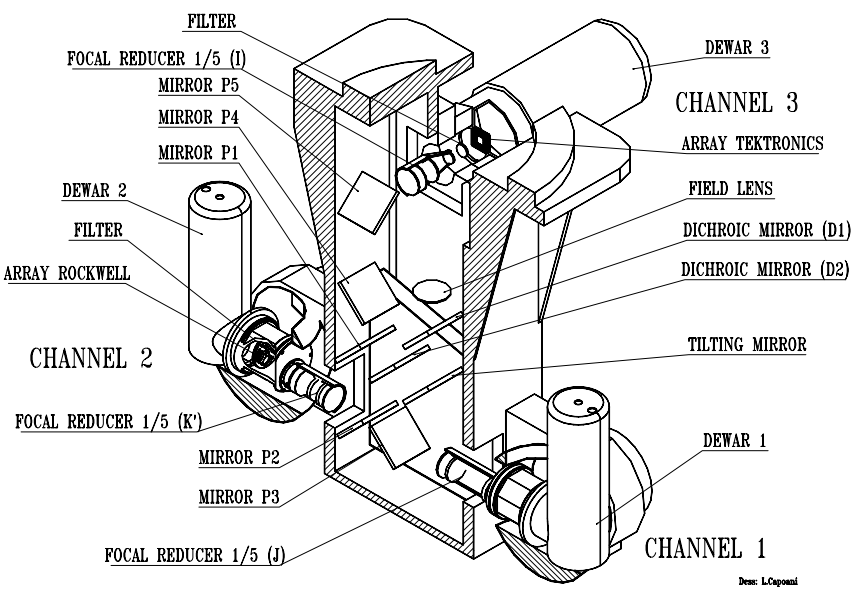

Fig. 1. Optics of the DENIS instrument

Photometric calibration is derived by observation of standard star fields. In order to compare our magnitude system to published ones, we need a precise definition of our photometric bands and an absolute calibration of the DENIS photometric system.

In Sect. 2, we describe the DENIS instrument and show the response curve for the complete system in the three bands. In Sect. 3, we estimate the conversion factors (ADUs to electrons) from the typical characteristics of DENIS images. Absolute calibration, based upon a synthetic Vega spectrum, is performed in Sect. 4, and observed and theoretical zero-points are compared.

\section{Instrument characteristics}

The DENIS instrument has been described in detail by Copet et al. (1999). A sketch of its main optical components is displayed in Fig. 1. It is located at the Cassegrain focus of the ESO $1 \mathrm{~m}$ telescope at La Silla Observatory (Chile). After reflection from the two telescope mirrors, the light beam goes through a field lens at the telescope focus, covered by a protective blade, both of $\mathrm{CaF}_{2}$ and uncoated. Then a dichroic splits the $i$ beam in reflection from the $J / K_{\mathrm{s}}$ beam in transmission.

The $i$ beam has two more reflections from coated mirrors before entering the objective $\left(3 \mathrm{CaF}_{2}\right.$ and 2 silica coated lenses), then goes through the Gunn $i$ filter, a shutter, the cryostat entrance window (BK7) and arrives at the Tektronix $1 \mathrm{~K}$ CCD detector, cooled to $180 \mathrm{~K}$.

The $J / K_{\mathrm{S}}$ beam is reflected off a microscanning mirror (uncoated $\mathrm{Al}$ ), then $J$ is reflected by a second dichroic and a coated mirror before entering the $J$ objective $\left(3 \mathrm{CaF}_{2}\right.$ and 2 fused silica coated lenses), then the cryostat entrance window (coated fused silica), the filter and finally the NICMOS-3 detector, both cooled to $80 \mathrm{~K}$.

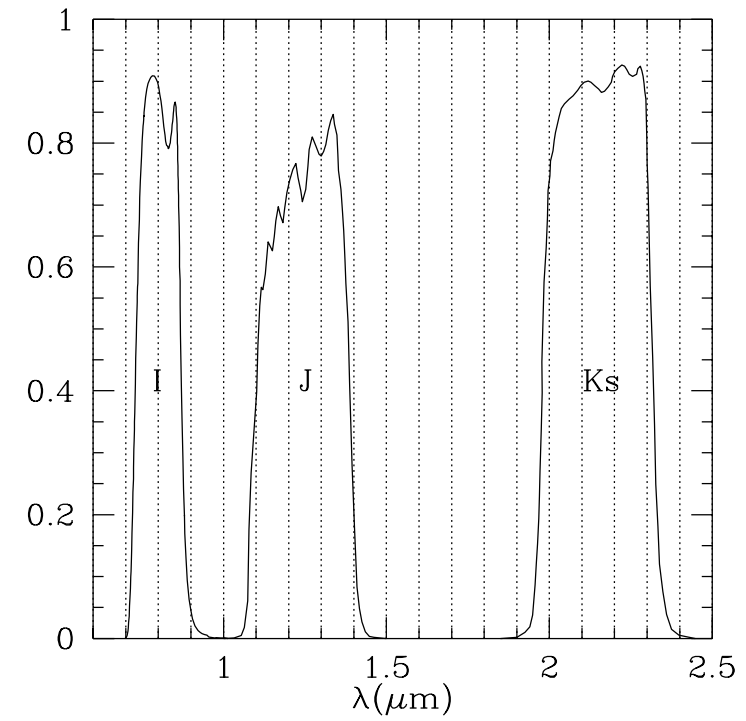

Fig. 2. The 3 DENIS filter response curves

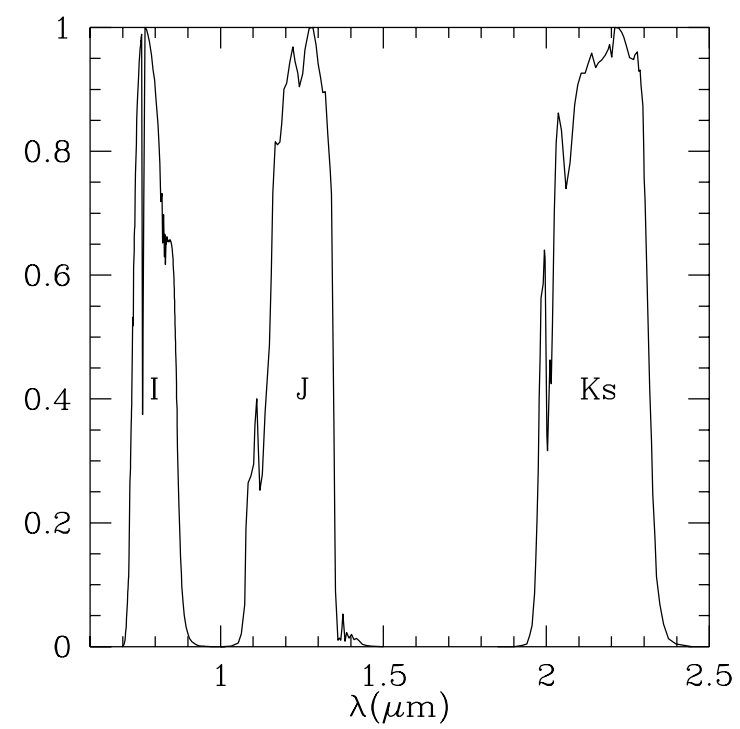

Fig. 3. The complete DENIS system normalized response curves (atmosphere, optics, filters, detectors)

The $K_{\mathrm{s}}$ beam is transmitted by the second dichroic, then reflects off two more coated mirrors, passes through the $K_{\mathrm{s}}$ objective ( $4 \mathrm{ZnS}$-Cleartran and 1 fused silica coated lenses), the cryostat entrance window (coated fused silica), the filter and the other NICMOS-3 detector.

We have tried to obtain response curves for all these elements. When this was not possible, a reasonable estimate of the transmission was adopted. Filter response curves as provided by Barr Associates (U.S.A.) for $J$ and $K_{\mathrm{s}}$, at the nominal detector temperature $(77 \mathrm{~K})$, and by MTO-France for the Gunn $i$ filter (at ambient temperature) are displayed in Fig. 2. The full system response curves (atmosphere, optics, filter and detector) are shown in Fig. 3. 
Table 1. Conversion factors (expected and measured, in $\left.\mathrm{e}^{-} / \mathrm{ADU}\right)$ in the three bands

\begin{tabular}{cccc}
\hline Gain & $i$ & $J$ & $K_{\mathrm{s}}$ \\
\hline Expected & 3.08 & 18.4 & 58.6 \\
Measured & 3.04 & 11.4 & 56.2 \\
Std dev & 0.01 & 0.1 & 0.1 \\
\hline
\end{tabular}

\section{Characteristics of DENIS images and conversion factors}

The first characteristics generally measured on astronomical images are the "sky emission" level (which includes telescope and instrument background in the $K_{\mathrm{s}}$ band), and the noise on sky images and darks.

Variations in sky level are observed in all three bands. In $i$, they are related to the distance to, and phase of, the Moon. In $J$, they are due to variations in the hydroxyl radical's emission intensity $\left(\mathrm{OH}^{-}\right.$Meinel bands), connected to the passage of density and temperature perturbations through the upper atmosphere (Ramsay et al. 1992). In $K_{\mathrm{s}}$ they come from the temperature variations. Also note that, in crowded fields, the background value is set by the confusion level (background of faint undistinguishable stars).

From these variations, the conversion factor between ADU (analog-to-digital units) and electrons can be deduced. Table 1 compares the result of these gain measurements with the expected values, calculated from the electronics characteristics of the chips, the preamplifiers gains, and the analog-to-digital conversions. Table 2 gives for each band the median value of the read-out noise in electrons, the median and faintest values of the sky level, in mag $\operatorname{arcsec}^{-2}$, and the median and minimal values of the sky image noise in electrons, calculated from more than 2000 images taken during the last year of observations (April 1998 to April 1999), and adopting zero-points of $23.5,21.3$, and 19.2 in $i, J$, and $K_{\mathrm{s}}$, respectively (see Sect. 4 and Table 5 ). The large values of sky and sky noise in $K_{\mathrm{s}}$ come from the thermal background of the instrument, which does not include cool stop optics. The "best" value of the sky level in $J$ is suspect.

Note that some care must be taken in applying the conversion factors and zero-points to the whole DENIS survey: first, $J$ and $K_{\mathrm{s}}$ conversion factors seem to be slightly variable ( 11.4 to 15.3 in $J, 51.6$ to 57.0 in $K_{\mathrm{s}}$ ), and second, changes in the instrument have altered these values: the pre-amplifier boards of $J$ and $K_{\mathrm{s}}$ cameras were changed in June 1996, and a spare $J$ camera has been in use from April 3, 1998 to May 9, 1999. Old conversion factors valid until June 1996 were 12.47 in $J$ and 39.3 in $K_{\mathrm{s}}$ (Chevallier 1996). Zero-point variations will be analyzed in a future paper.
Table 2. Read-out noise, sky level, and sky image noise in the three bands

\begin{tabular}{cccccc}
\hline \multirow{2}{*}{ Band } & \multirow{2}{*}{ RON } & \multicolumn{2}{c}{ SKY } & \multicolumn{2}{c}{ SKY noise } \\
\cline { 3 - 6 } & $\mathrm{e}^{-}$ & $\begin{array}{c}\text { median } \\
\text { mag } \operatorname{arcsec}^{-2}\end{array}$ & $\begin{array}{c}\text { best } \\
\text { mag } \operatorname{arcsec}^{-2}\end{array}$ & $\begin{array}{c}\text { median } \\
\mathrm{e}^{-}\end{array}$ & $\begin{array}{c}\text { min } \\
\mathrm{e}^{-}\end{array}$ \\
\hline$i$ & 7.3 & 19.2 & 20.2 & 15 & 11 \\
$J$ & 21 & 16.6 & $18.1 ?$ & 37 & 27 \\
$K_{\mathrm{s}}$ & 40 & 11.2 & 12.0 & 311 & 222 \\
\hline
\end{tabular}

Table 3. Mean filter wavelengths (pure filter, and filter + atmosphere + detector, hereafter "fad"), effective and isophotal wavelengths for Vega

\begin{tabular}{cccc}
\hline Wavelength $(\mu \mathrm{m})$ & $i$ & $J$ & $K_{\mathrm{s}}$ \\
\hline$\lambda_{\text {fil }}$ & 0.802 & 1.248 & 2.152 \\
$\lambda_{\text {fad }}$ & 0.795 & 1.235 & 2.160 \\
$\lambda_{\text {eff }}$ & 0.788 & 1.221 & 2.144 \\
$\lambda_{\text {iso }}$ & 0.791 & 1.228 & 2.145 \\
\hline
\end{tabular}

Table 4. Flux densities of a zero magnitude star for the three DENIS bands

\begin{tabular}{cccc}
\hline Band & $\begin{array}{c}\lambda_{\text {iso }} \\
\mu \mathrm{m}\end{array}$ & $\begin{array}{c}F_{\lambda} \\
\mathrm{W} / \mathrm{m}^{2} / \mu \mathrm{m}\end{array}$ & $\begin{array}{c}F_{\nu} \\
\mathrm{Jy}\end{array}$ \\
\hline$i$ & 0.791 & $1.2010^{-8}$ & 2499 \\
$J$ & 1.228 & $3.1710^{-9}$ & 1595 \\
$K_{\mathrm{s}}$ & 2.145 & $4.3410^{-10}$ & 665 \\
\hline
\end{tabular}

\section{Absolute calibration}

The next interesting characteristics to establish for absolute photometric work is the flux of a zero magnitude star in the three DENIS bands. Many infrared systems and several ways to calibrate them exist. We have decided to use the calibration scheme described by Cohen et al. (1992): they start from a model of Vega from Kurucz (1991), taking into account its lower than solar metallicity, and normalize it to $F_{5556}=3.4410^{-8} \mathrm{~W} \mathrm{~m}^{-2} \mu \mathrm{m}^{-1}$ from Hayes (1985). Additionally, we adopt $V=0.03 \mathrm{mag}, V-I=0$, so $I=0.03 \mathrm{mag}$, but $J H K L M=0.00 \mathrm{mag}$ for this star. For a more detailed discussion of Vega magnitudes and colours, see Bessell et al. (1998).

We must now determine the isophotal wavelength of each filter, taking into account the filter response curve, the atmospheric transmission, the detector radiance response and the Vega spectrum. Isophotal wavelengths are preferred over effective wavelengths, because the latter vary with input source spectrum much more than do the former (see Golay 1974, for details and definitions). Results are given in Table 3.

Using the isophotal wavelengths of our bands, and the Vega spectrum, we can compute the flux densities for a zero magnitude star. Table 4 gives the results in wavelength and frequency units. 
Table 5. Theoretical and observed zero-points. Derived overall transmission and its components $(\tau, \rho$ and $Q E$ correspond to transmission, reflection and quantum efficiency, respectively)

\begin{tabular}{cccc}
\hline Parameter & $i$ & $J$ & $K_{\mathrm{s}}$ \\
\hline Theoretical zero-point & 25.10 & 23.29 & 20.43 \\
Observed zero-point $\left(7^{\prime \prime}\right)$ & 23.4 & 21.1 & 19.1 \\
Corrected zero-point & 23.5 & 21.3 & 19.2 \\
Measured overall transmission & 0.24 & 0.16 & 0.32 \\
\hline$\tau_{\text {atmosphere }}$ & 0.955 & 0.912 & 0.912 \\
$\tau_{\text {mirrors }}$ & $0.868^{2}$ & $0.964^{3}$ & $0.978^{3}$ \\
$\tau_{\text {blade, field lens }}$ & $0.938^{2}$ & $0.939^{2}$ & $0.940^{2}$ \\
$\tau_{\text {dichroics }}$ & & 0.784 & $0.810 \times 0.870$ \\
$\rho_{\text {dichroics }}$ & 0.993 & 0.970 & \\
$\tau_{\text {coated mirrors }}$ & $0.993^{2}$ & 0.970 & $0.97^{2}$ \\
$\tau_{\text {objective }}$ & $0.979^{3} \times 0.982^{2}$ & $0.979^{3} \times 0.985^{2}$ & $0.70 \times 0.982$ \\
$\tau_{\text {cryostat window }}$ & 0.94 & 0.985 & 0.982 \\
$\tau_{\text {filter }}$ & 0.909 & 0.846 & 0.926 \\
$Q E_{\text {detector }}$ & 0.65 & 0.8 & 0.8 \\
\hline Resulting overall transmission & 0.31 & 0.32 & 0.25 \\
\hline
\end{tabular}

From these flux densities, we can estimate how many ADUs would be measured if the atmosphere, telescope and instrument totally transmitted the photons from this zero magnitude star. Comparing to the actually observed zeropoint will give the overall transmission of the system. We first integrate the product of the Vega spectrum (shifted by $0.03 \mathrm{mag}$ in $i$ ) by the transmission of the full system (fad), over the wavelength domain of our filters $\left(\lambda_{0}\right.$ and $\lambda_{1}$ correspond to the first and last wavelengths where filter transmission reaches 0 ), to obtain the measured flux of a zero magnitude star:

$F_{\mathrm{t}}=\int_{\lambda_{0}}^{\lambda_{1}} S(\lambda) \frac{F_{\lambda}(\text { Vega })}{h \nu} \mathrm{d} \lambda$.

The theoretical zero-point is given by:

$Z P_{\mathrm{th}}=2.5 \log \left(F_{\mathrm{t}} \times A t / G\right)$,

where $A$ is the unobscured telescope collecting area $\left(0.68 \mathrm{~m}^{2}\right), t$ is the effective integration time $(8.998 \mathrm{~s}$ in $i, 8.809 \mathrm{~s}$ in $J$ and $K_{\mathrm{s}}$ ), and $G$ is the conversion factor. Table 5 gives the results.

Zero-points are measured during calibration nights, where only photometric standards are observed, and routinely during survey nights, to follow possible instrumental variations and make a rough estimate of the extinction coefficients. They are measured from aperture magnitudes inside a 7 arcsec diameter circle around the standard star. To make a valid comparison with the theoretical zero-points, a first correction is necessary to include flux falling outside this aperture. This has been estimated to amount to $0.1 \mathrm{mag}$ in all three bands from a comparison of observations through a 15 arcsec diameter aperture.

A linear fit of the observed magnitudes vs. airmass (assuming that Bouguer's 1729, law is valid) gives the extinction coefficient as the slope and the zero-point as the intercept. However, it is well known that extrapolation to zero airmass leads to a systematic error in the nearinfrared (the Forbes 1842, effect), which has been quantified for the $J$ and $K$ bands by Manduca \& Bell (1979). For the La Silla typical water vapour contents (1 to $10 \mathrm{~mm}$ of precipitable water), the error is about $0.10 \mathrm{mag}$ in $J$ and $0.02 \mathrm{mag}$ in $K$, and should be similar in $K_{\mathrm{s}}$. Therefore, we also add this $J$ correction to the observed zero-point, while we neglect the $K$ correction, given the uncertainty in measured zero-points. The corrected zero-points (for infinite aperture and non-linear variation with airmass) are given in Table 5, and the derived overall transmissions follow.

To interpret the measured overall transmission, we have tried to estimate the contribution of each component of the system, namely atmosphere, aluminium reflections (telescope mirrors, and microscanning mirror for $J$ and $K_{\mathrm{s}}$ ), thin blade protecting the field lens, field lens itself, dichroics, coated mirrors, objectives, cryostat entrance windows, filters, and detector quantum efficiency (converted to radiance response). For details about each value, see Galliano (1999). Table 5 gives all these estimates, and their final product. The agreement with the measured overall transmission is satisfying, and shows a good performance of the instrument, with overall throughput of 20 to $30 \%$ in all three bands.

Acknowledgements. Thanks to Maria Eugenia Gómez for finding the original reference of Bouguer's law.

The DENIS project is supported by the SCIENCE and the Human Capital and Mobility plans of the European Commission under grants CT920791 and CT940627, by the French Institut National des Sciences de l'Univers, the Ministère de l'Éducation Nationale and the Centre National de la Recherche Scientifique, in Germany by the State of Baden-Würtemberg, in Spain by the DGICYT, in Italy by the Consiglio Nazionale delle Ricerche, by the Austrian Fonds zur Förderung der wissenschaftlichen Forschung und 
Bundesministerium für Wissenschaft und Forschung, in Brazil by the Fundation for the development of Scientific Research of the State of São Paulo (FAPESP), and by the Hungarian OTKA grants F-4239 and F-013990, and the ESO C \& EE grant A-04-046.

\section{References}

Bessell M.S., Castelli F., Plez B., 1998, A\&A 333, 231 (and erratum in A\&A 337, 321)

Bouguer P., 1729, Essai d'optique sur la gradation de la lumière, C. Jombert, Paris, reprinted in: Les maîtres de la pensée scientifique, Paris, 1921

Chevallier L., 1996, Performances et qualité d'images du survey DENIS, DEA (master's) thesis. University of Strasbourg

Cohen M., Walker R.G., Barlow M.J., Deacon J.R., 1992, AJ 104, 1650

Copet E., Epchtein N., Rouan D., et al., 1999, A\&AS (in preparation)

Epchtein N., de Batz B., Copet E., et al., 1994, in: Proceedings of a Conference held at Les Houches: "Science with astronomical near-infrared sky surveys", Epchtein N., Omont A., Burton W.B., Persi P. (eds.), "A Deep NearInfrared Survey of the Southern Sky", september 1993, p. 3, Les Houches, France, reprinted from Astrophysics and Space Science 217, 3

Forbes J.D., 1842, Phil. Trans. 132, 225

Galliano E., 1999, Une calibration absolue du système photométrique du survey DENIS, DEA (master's) thesis. University of Grenoble

Golay M., 1974, in: Introduction to astronomical photometry. Reidel, Dordrecht, p. 39

Hayes D.S., 1985, in: Calibration of Fundamental Stellar Quantities, Proc. IAU Symposium No. 111, Hayes D.S., Pasinetti L.E. \& Davis Philip A.G. (eds.). Reidel, Dordrecht, p. 225

Kurucz R.L., 1991, quoted in Cohen et al. (1992) (as private communication)

Manduca A., Bell R.A., 1979, PASP 91, 848

Ramsay S.K., Mountain C.M., Geballe T.R., 1992, MNRAS 259,751 